\title{
Wavelet thresholding method based on hypotheses testing and noise estimation for multipath mitigation in GPS receivers
}

\author{
Vahid Alipour Maralani, Chenxi Huangfu, Yun Zhao \\ School of Electronic and Information Engineering \\ Beihang University \\ Beijing, P.R.China
}

\begin{abstract}
Urban navigation applications of the global positioning system (GPS) suffer from the problem of multipath propagation caused by complex signal reflections in local environments. The resulting errors of ranging can finally lead to an unacceptable degradation of positioning accuracy for many GPS applications. To mitigate the multipath effects in GPS receivers, an approach based on a hypotheses testing wavelet thresholding filter and the real-time noise estimation is proposed. Integrated into the code and carrier tracking loops of GPS receivers, this technique extracts the multipath tracking errors, which are applied directly to compensate the pseudorange and carrier-phase measurements due to the multipath effect. Simulation results indicate that under a fairly severe multipath scenario in urban navigation environments, the proposed method achieves the improvement of the positioning accuracy at the expense of a little additional computation load.
\end{abstract}

Keywords-global positioning system (GPS); multipath mitigation; wavelet thresholding filter; hypotheses tesing; multipath tracking errors

\section{INTRODUCTION}

Despite the continuous progress in global positioning system (GPS) receivers, the error due to multipath propagation has remained an obstacle to accuracy improvement [1]. The multipath propagtion is a serious challenge, especially in urban environments where a large number of reflectors for the satellite navigation signals exist, such as high buildings. The resulting degradation of positioning accuracy cannot be considered as insignificant for many GPS applications. Measuring the propagation time of the navigation signal from the GPS satellites to a user's receiver of allows estimation of the user's position, and the corresponding measurement errors caused by the multipath propagation can introduce a positioning error to the receiver up to a hundred of meters [2]. Whereas for high-precision applications, the resulting carrier phase error could be orders of magnitude higher than that of a multipath-free case [3].

Conventional GPS receivers provide no inherent discrimination against multipath propagation. The reception of additional navigation signal replica due to multipath propagation introduces a bias error into the time-delay estimate of the code delay lock loop (DLL) and also affects the carrier phase-locked loop (PLL), which finally leads to a bias error in the estimates of the user's position.

To reduce the multipath effects from the perspective of the receiver's baseband processing, several DLL-based methods have been proposed, such as the strobe correlator [4] or pulse aperture correlator [5]. This family of methods achieves a modified discriminator function. Their advantages are the simple implementation and low computational load. Nevertheless, a major disadvantage is that it reduces the tracking capability of the DLL. Multipath estimation methods, such as the multipath estimation DLL [6] or multipath mitigation technique [7], applies the maximum-likelihood estimation theory to estimate the parameters of the multipath components. However, they are only suitable for quasi-static multipath scenarios. Multipath estimators based on the sequential Bayesian estimation [8], require the state transition models of the multipath process as the first step towards dynamic estimation. Unfortunately, for complex multipath environments, obtaining the prior multipath models is virtually impossible. Without the need to assume multipath models, the adaptive filtering techniques offer the possibilities of different solution, which have been addressed in some literatures. In Ref. [3], a wavelet filter based on the conventional thresholding method was proposed to mitigate the GPS mutlipath effect in a receiver with a fixed trajectory movement. Since the involved application is rare, it's not clear that this filter is applicable to common GPS applications.

In this paper, we propose a multipath mitigation approach for GPS receivers on the basis of the hypotheses testing wavelet thresholding filter and a real-time noise estimation technique. This paper is organised as follows. Section 2 outlines how the wavelet filtering technique is applied to the GPS receiver for multipath mitigation. Section 3 describes the multipath effect on the tracking loops of GPS receivers. Section 4 presents the wavelet thresholding filter based on hypotheses testing and noise estimation. Section 5 demonstrates the simulation results under a fairly severe multipath urban scenario. Section 6 concludes this paper.

\section{WAVELET Filtering FOR MULitPath Mitigation IN GPS RECEIVERS}

From the point of view of wavelet analysis, the signal 
component and noise have different Lipschitz exponents [9] at the wavelet multi-scale levels, and therefore they can be separated from each other. According to Ref. [3], the multipath errors and observation noise have distinct frequency properties, i.e. low-frequency and high-frequency respectively. In the case of GPS applications, noise has a typical property of highfrequency, while the navigation signals arriving at the receivers have a typical property of low-frequency, and the multipath errors have the frequency property between the typical high-frequency and the typical low-frequency. Thus, it can be concluded that the multipath errors can be separated from observation noise or navigation signal components by using a correctly designed wavelet filter.

The proposed wavelet filtering approach for multipath mitigation integrated into one receiver tracking channel is illustrated in Fig. 1. For this approach, the outputs of the code and the carrier-phase discriminators are used as the inputs. The wavelet filter requires a known variance of the observation noise, which should be previously estimated. The extracted multipath tracking errors as the outputs are subsequently applied directly to compensate for the multipath effect both in pseudorange and carrier-phase measurements, and then the improvement in the position accuracy can be achieved.

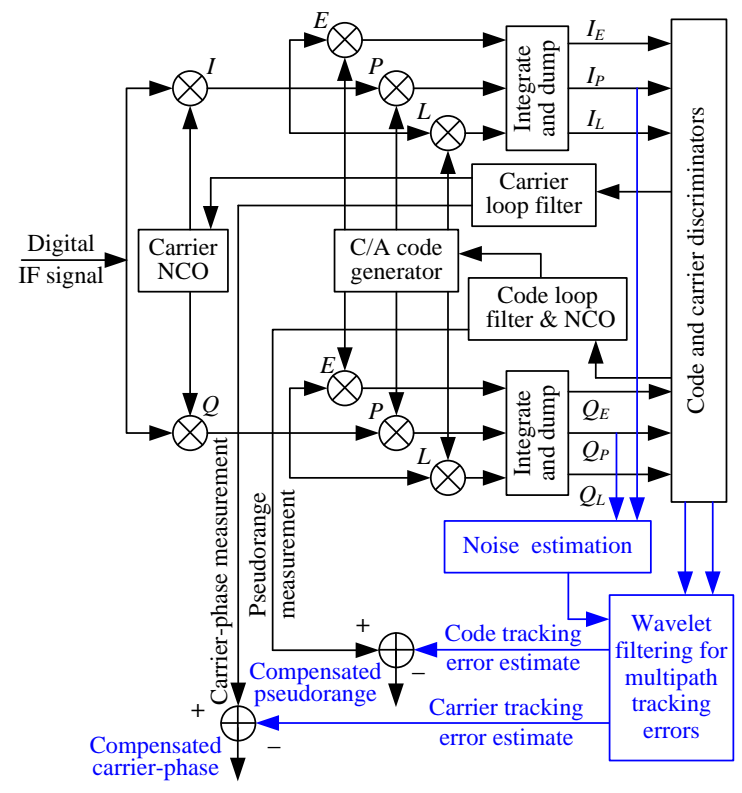

Fig.1. Wavelet-based multipath mitigation in a GPS receiver tracking channel.

\section{MUlTIPATH EFFECT ON CODE OR CARRIER TRACKING PERFORMANCE}

In the multipath case, after the front-end processing and correlation with the locally generated signal of the receiver, the resulting in-phase $(I)$ components of a pair of early and late correlator outputs, i.e. $R_{E I}(\tau)$ and $R_{L I}(\tau)$, can be expressed as

$$
\begin{aligned}
& R_{E I}(\tau)=A_{c} R_{c}\left(\tau-\tau_{d}\right) \cos \theta+\sum_{n=1}^{N} \alpha_{n} A_{c} R_{c}\left(\tau-\tau_{d}-\tau_{n}\right) \cos \left(\theta_{m_{n}}-\theta\right) \\
& R_{L}(\tau)=A_{c} R_{c}\left(\tau+\tau_{d}\right) \cos \theta+\sum^{N} \alpha_{n} A_{c} R_{c}\left(\tau+\tau_{d}-\tau_{n}\right) \cos \left(\theta_{m_{n}}-\theta\right)
\end{aligned}
$$

where $A_{c}$ is the received ${ }^{n=1}$ signal amplitude, $R_{c}(\cdot)$ is the correlation function of the spread-spectrum code (i.e., C/A code) sequence, $\tau$ represents the code delay estimated by the receiver, which consists of the LOS signal propagation delay estimation and the code tracking error caused by the multipath; $\tau_{d}$ is the correlation spacing for the $E$ and $L$ correlators; $\theta$ represents the estimated carrier phase, which consists of the LOS signal carrier phase estimation and the carrier phase tracking error caused by the multipath; $\left\{\alpha_{1}, \alpha_{2}, \ldots, \alpha_{N}\right\}$ represents the multipath amplitude ratio with respect to the LOS signal, $\left\{\tau_{1}, \tau_{2}, \ldots, \tau_{N}\right\}$ is the multipath delay vector, and $\left\{\theta_{m 1}, \theta_{m 2}, \ldots, \theta_{m N}\right\}$ is the multipath phase vector..

The corresponding quadrature-phase $(Q)$ components of the correlation output can be obtained by replacing the cosine function in the above equations with the sine function.

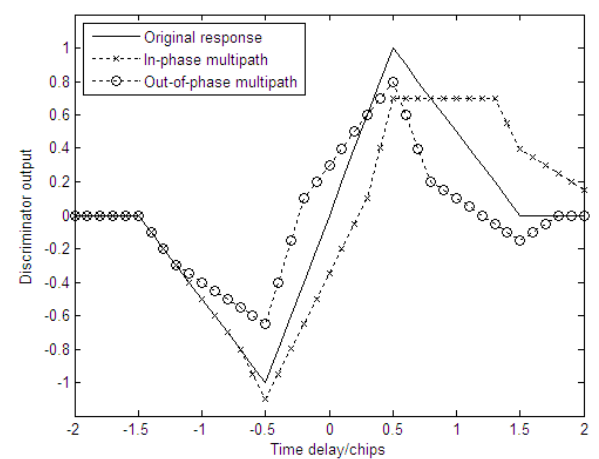

Fig. 2. DLL discriminator response due to multipath effect.

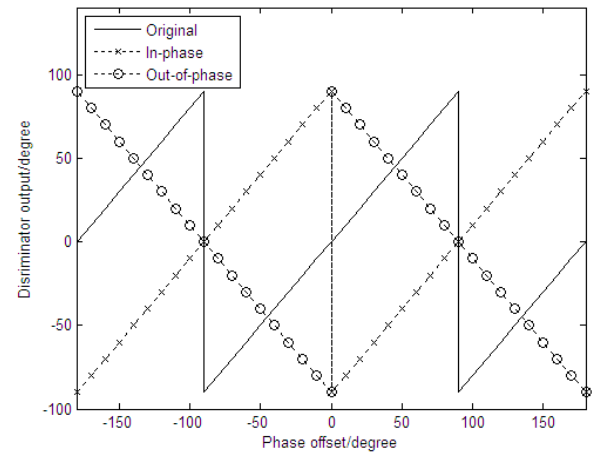

Fig. 3. PLL discriminator response due to multipath effect.

For the conventional receiver tracking loops, the DLL operation is based on the measurement of the early $(E)$ and late $(L)$ correlator outputs. The correlation function distorted by the multipath effect will result in an additional code tracking error. Fig. 2 shows the multipath response of a standard non-coherent early-minus-late DLL discriminator [10], where only one multipath component is considered, which is just in-phase or out-of-phase with the direct signal. The solid line represents the ideal discriminator response, the dotted line with cross markers represents the distorted response due to the in-phase multipath component, and the dotted line marked with circles indicates the distorted response due to the out-of-phase multipath component. Because the carrier phase estimation depends on the in- and quadraturephase components of the prompt correlation output, an additional phase tracking error is also introduced. Fig. 3 shows the multipath response of a standard actan PLL discriminator [10]. Obviously, the outputs of the PLL discriminator in the 
multipath case represented by the dotted line and the dotted line marked with circles are far less likely than the ideal output represented by the solid line. Meanwhile, the noise from the satellite channel and the receiver itself comes with the code or carrier multipath error in actual applications, which makes separating them difficult.

\section{WAVELET THRESHOLDING FILTER BASED ON HYPOTHESES TESTING AND NOISE ESTIMATION}

The multipath error and the observation noise exit in the DLL or the PLL output in the form of an aliasing (mixing) observation. The wavelet thresholding filtering is then employed in this paper in consideration of the computational efficiency of this family of methods [11]. The wavelet transform and the multi-scale decomposition by using the Mallat fast algorithm [9] can provide the approximation of a signal $f(t)$ at the $j$ th scale, which can be defined as

$$
f(t)=\sum_{k} c_{j k} 2^{-j / 2} \Phi\left(2^{-j} t-k\right)+\sum_{k} d_{j k} \psi\left(2^{-j} t-k\right)
$$

where $\Phi(t)$ and $\psi(t)$ are the scaling function and the wavelet function, respectively; $c_{j k}$ and $d_{j k}$ are the $k$ th scaling and wavelet coefficients separately at the $j$ th scale, and they can be derived by the scaling coefficients at the $(j-1)$ th scale, which can be expressed as

$$
\begin{aligned}
c_{j k} & =\sum_{l} h_{l-2 k} c_{(j-1) l} \\
d_{j k} & =\sum_{l} g_{l-2 k} c_{(j-1) l}
\end{aligned}
$$

where $h_{l}$ and $g_{l}$ represent the $l$ th element of the coefficients of the high-pass and the low-pass filter at the $(j-1)$ th scale, respectively, which are dependent on $\Phi(t)$ and $\psi(t)$. Whereas for the signal reconstruction, the inverse algorithm of the above transform is then used.

There are many options about the wavelet bases, though the appropriate choice depends upon the specific application. In order to obtain a good symmetrical property, we choose the Symmlets compactly supported wavelet base [9], and its vanishing moments is set to be 8 . Regarding the levels (or scales) of the wavelet decomposition, we choose 5 levels.

Aiming at the removal of the noise in a signal, the wavelet thresholding approaches are implemented by thresholding wavelet coefficients. Thus, when the reconstruction by inverse wavelet transform is performed, noise (undesired component) is removed or at least reduced. In the case of this paper, the remained component (desired component) is relative to the multipath code or carrier tracking error.

The choice of threshold involved in the wavelet thresholding approaches is a crucial and tricky issue. The threshold must be chosen just above the maximum level of the noise. It has been proved that the maximum amplitude of the noise has a very probability of being just below the Donoho threshold [9], [12]. The Donoho universal threshold is defined as

$$
\lambda=\sigma \sqrt{2 \log _{\mathrm{e}}(L)}
$$

where $\lambda$ represents the threshold, $\sigma$ is the observation noise level (i.e. standard deviation), $L$ is the length of the noisy sequence. However, the estimation of noise level in actual applications is rather difficult, and then an empirical noise estimator is widely used (e.g. in Ref. [3]) and given by

$$
\hat{\sigma}=\operatorname{median}\left\{\left|c D_{J k}\right|\right\} / 0.6745
$$

where $c D_{J k}$ are the wavelet coefficients at the finest scale $J$, the subscript $k$ represents the $k$ th element, median $(\cdot)$ is the median function, and then its output is the median of the absolute value of $c D_{J k}$. 0.6745 is a empirical constant.

Ref. [9] points out that the Donoho threshold is not optimal, and generally it is a higher threshold. Therefore, in this paper, a lower threshold will be determined by using hypotheses testing. Further, in order to make the good estimation of noise, the empirical noise estimator in Eq. (7) is replaced by a real-time noise estimator by using the actual correlation values in GPS receivers. In detail, the noise level is estimated by taking the standard deviation of the correlation values out-of-1-chip early with respect to the correlation peak in the prompt correlator. Since the multipath components are delayed with respect to the LOS component, the correlation values for out-of-1-chip early time delays are not affected any multipath components [13]. This concept is explained by Fig. 4. Furthermore, for dynamic navigation applications, the observation noise is time-varying, and then a short-time data window (10ms is set in this paper) is chosen for real-time processing. This indicates the time-varying noise level $\sigma(t)$, and also the time-varying threshold $\lambda(t)$.

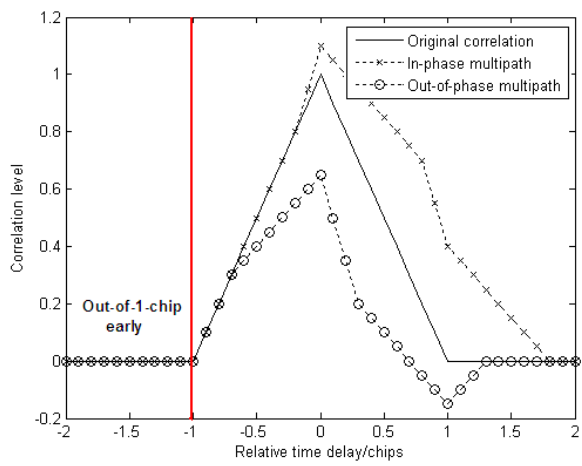

Fig. 4. Noise estimation by taking out-of-1-chip early correlation values

From a statistical point of view, thresholding is closely related to hypotheses testing, and the latter one can control false discovery rate (FDR) [11], [14]. The FDR is the expected percentage of false predictions in the set of predications, and in this paper, it refers to the probability of the observation noise is incorrectly separated by wavelet thresholding. Regarding the wavelet thresholding procedure, for each data window (i.e.10ms), the following 5 steps are carried out to determine the threshold on hypotheses testing and threshold the wavelet coefficients

Step 1. Calculate the following probability for each wavelet coefficient $c D_{j k}(t)$ obtained by the decomposition with five levels:

$$
p_{j k}(t)=2\left[1-\Phi\left(\left|c D_{j k}(t)\right| / \sigma(t)\right)\right]
$$

where $\Phi(\cdot)$ represents the normal distribution function, and $\sigma(t)$ is the standard deviation of the observation noise within the data window. taken from the correlation values out-of-1chip early from the prompt correlator.

Step 2. Sort all the probability values obtained in Step 1 in ascending order as

$$
p_{(1)} \leq p_{(2)} \leq \ldots . . \leq p_{(m)}
$$

where $p_{j k}(t)$ is replaced by $p_{(i)}$, and the subscript $i$ represents the $i$ th element of the sequence consisting of $m$ elements. 
Step 3. Calculate the critical value $c_{(i)}=(i / m) q$ for $p_{(i)}, q$ is the FDR, and set to be 0.05 in this paper. Further, do testing: if $p_{(i)}$ $\leq c_{(i)}$, then the desired FDR is achieved and let the largest $i$ be $i_{0}$ :

$$
i_{0}=\max \left\{i \mid p_{(i)} \leq c_{(i)}\right\}
$$

Step 4. On the basis of $p_{(i 0)}$, carry out the inverse computation of the Eq. (8), then the threshold is determined by:

$$
\lambda(t)=\sigma(t) \Phi^{-1}\left(1-p_{(i 0)} / 2\right)
$$

Step 5. Threshold all the wavelet coefficients with $\lambda(t)$ : for each wavelet coefficient, compare it with the threshold, if $c D_{j k}$ $(t) \geq \lambda(t)$, then the wavelet coefficient is retained; otherwise, the wavelet coefficient is replaced by a zero. Then, all the wavelet coefficients are used for the wavelet reconstruction, and then the noise is removed.

\section{SimUlation RESUlts}

For the performance assessment, simulations using the proposed multipath equalisation method are carried out. The multipath signals are generated by a satellite navigation signal simulator [15], which provides multipath simulation functions for urban navigation environments. In the simulations, a typical multipath scenario shown in Fig. 5 is specified in which a vehicle carrying a receiver with the receiving antenna mounted on its roof moves along a road flanked by high buildings and has a straight-line trajectory at a speed of $10 \mathrm{~m} / \mathrm{s}$. Compared with the straight line from the sky which represents the LOS signal, the fold lines in Fig. 5 indicate the multipath components. There are many fold lines, which indicates this is a fairly severe multipath scenario. Embedded in the proposed wavelet threshoding method, a software GPS receiver [16] processes the above simulated signals.

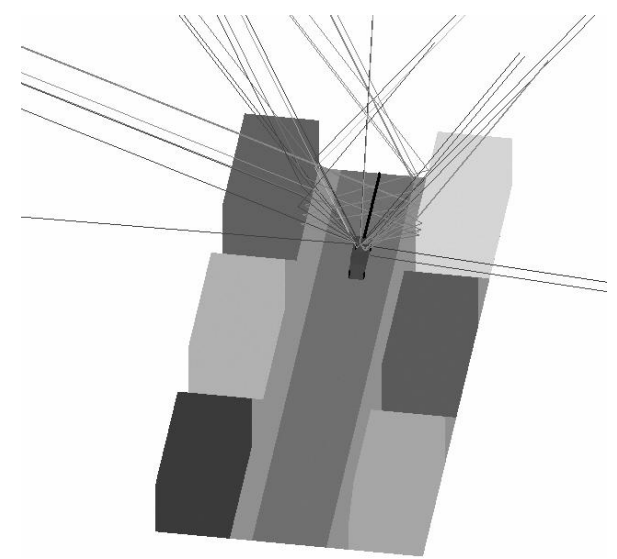

Fig. 5. Simulated multipath scenario in urban environment.

Fig. 6(a) and (b) show the multipath code tracking error and the multipath carrier-phase tracking error (the black lines in Fig. 6) extracted by using the proposed wavelet threshoding approach respectively, which are compared with the corresponding errors (the gray lines in Fig. 6) extracted by using the conventional wavelet thresholding approach. It is indicated in Fig. 6 that the threshold determined by the conventional wavelet threshoding method is generally higher. The multipath tracking errors are subsequently applied directly to compensate for the multipath effect in the receiver's measurements and positioning results. The horizontal positioning errors for the proposed wavelet threshoding approach (the black line in Fig. 7) and the conventional wavelet threshoding method (the thick gray line in Fig. 7) are shown in Fig. 7, and they are compared with the standard receiver processing result (the thin gray line in Fig. 7). The corresponding positioning errors are $1.75 \mathrm{~m}(1 \sigma), 2.20 \mathrm{~m}(1 \sigma)$ and $15.95 \mathrm{~m}(1 \sigma)$, respectively. And the proposed wavelet threshoding method achieves the biggest improvement of the positioning accuracy.

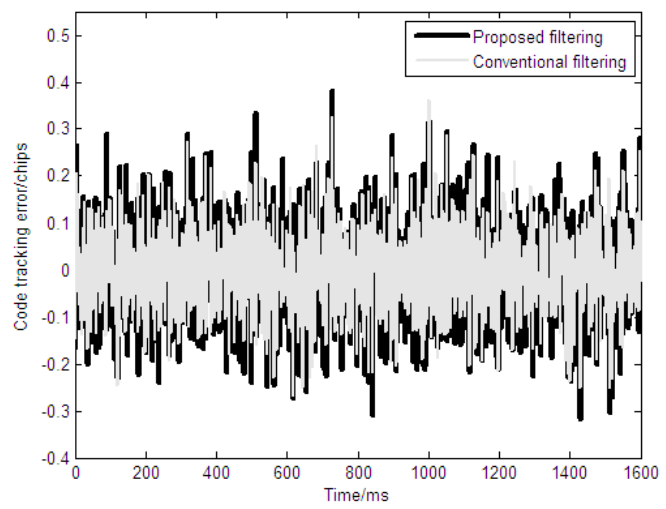

(a) ultipath code tracking error

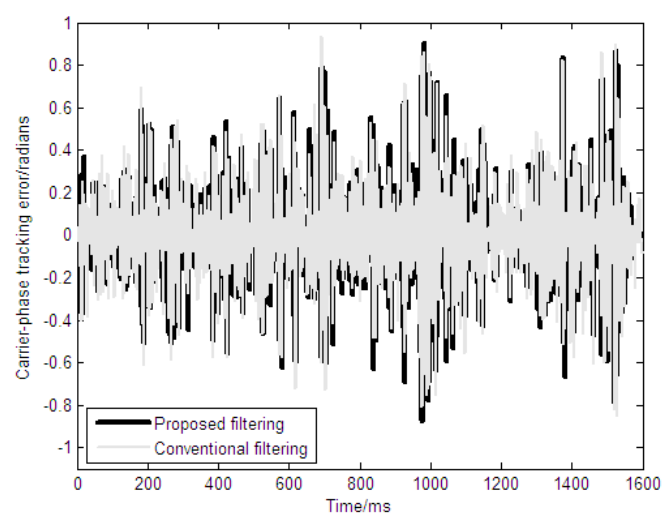

(b) Multipath carrier phase tracking error

Fig. 6. Multipath errors reconstructed by the wavelet thresholding filter.

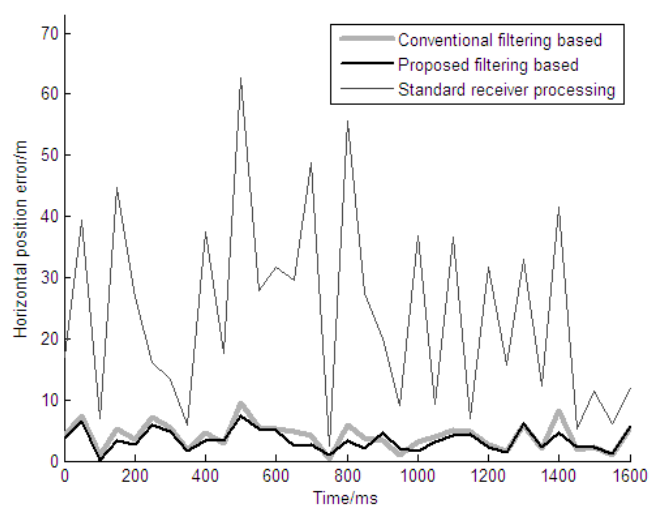

Fig. 7. Positioning performance of the wavelet thresholding filter.

\section{CONCLUSIONS}

A baseband-processing-based multipath mitigation approach for simultaneous estimation of the multipath tracking errors and compensation of the multipath effect in the 
receiver's measurements and positioning results has been proposed in this paper, which is achieved by applying the wavelet thresholding technique based on hypotheses testing and a real-time noise estimator. Compared with the conventional wavelet threshoding method, the accurate noise estimation on the basis of the actual correlation values in the receiver replaces the empirical noise estimation, and a timevarying and more accurate threshold is determined by using hypotheses testing procedure.

To evaluate the performance, simulations that employ a navigation satellite signal simulator and a GPS software receiver are carried out. Under a fairly severe multipath scenario in urban navigation environments, the proposed method achieves the improvement of positioning accuracy, in comparison with the conventional wavelet threshoding method and also the standard receiver processing. While compared with other baseband-processing-based multipath mitigation methods, the proposed method requires no prior information, and a little additional computation load due to no recursive algorithm involved.

\section{ACKNOWLEDGMENT}

This research was supported by the National Natural Science Foundation of China (No. 61101075).

\section{REFERENCES}

[1] X. Feng, X. Wu and Z. Zhang, "Multipath mitigation technique based on modifications to GNSS monitor station antennas field", CSNC 2012 Proceedings. Berlin: Springer-Verlag, 2012, pp. 655-666.

[2] P. Closas, C. Fernandez-Prades and J. A. Fernandez-Rubio, "A Bayesian approach to multipath mitigation in GNSS receivers" , IEEE Journal of Selected Topics in Signal Processing, vol. 3, no.4, pp. 695-706, 2009.

[3] E. M. Souza, J. F. Monico and W. G. Polezel, "Spectral analysis and lowfrequency multipath mitigation for kinematic applications", 2008
IEEE/ION Position, Location and Navigation Symposium. Seattle: Omnipress, 2008, pp. 413-417.

[4] L. Garin, F. Diggelen and J M Rousseau, "Strobe and edge correlator multipath mitigation for code", ION GPS-96 Proceedings. Manassas: ION, 1996, pp. 657-664.

[5] J. Jones, P. Fenton and B. Smith, "Theory and performance of the pulse aperture correlator", Available from: http://www.novatel.com/ Documents/Papers/PAC.pdf.

[6] B. R,.Townsend, P. C. Fenton and K. J. Dierendonck, "L1 carrier phase multipath error reduction using MEDLL technology", ION GPS-96 Proceedings. Manassas: ION, 1995, pp.1539-1544.

[7] P. C. Fenton and J. Jones, "The theory and performance of NovAtel Inc.'s vision correlator", ION GNSS 2005 Proceedings. Manassas: ION, 2005, pp. 2178-2186.

[8] M. Lentmaier, B. Krach and P. Robertson, "Bayesian time delay estimation of GNSS signals in dynamic multipath environment", International Journal of Navigation and Observation: Special Issue on Future GNSS Signals, pp. 1-11, 2008.

[9] S. Mallat, A Wavelet Tour of Signal Processing: The Sparse Way. 3rd ed. Singapore: Elsevier Inc, 2009.

[10] E.D. Kaplan and C. J. Hegarty. Understanding GPS: principles and applications. 2nd ed. Boston: Artech House, 2006.

[11] Q. Pang and L. Zhang, Wavelet Filtering Methods and Applications. Beijing: Tsinghua University Press, 2005.

[12] D. L. Donoho, "De-noising by soft-thresholding", IEEE Trans. Inform. Theory, vol. 41, no.3, pp. 613-627, 1995.

[13] M. Z. Bhuiyan, E. S. Lohan, "Advanced multipath techniques for satellite-based positioning applications", International Journal of Navigation and Observation, 15 pages, Article ID 412393, 2010.

[14] W. Du, C. Liu and Y. Li, "Wavelet threshold de-noising based on multiple hypothesis test", Journal of Vibration and Shock, vol. 30, no. 7, pp. 197200, 2011.

[15] Y. Zhao and Y. Kou, "Multi-constellation GNSS signal simulator and its software implementation", ION GNSS 2009 Proceedings. Manassas: ION, 2009, pp. 2213-2219.

[16] Y. Zhao and Q. Zhang, "Architecture of software GPS receiver and implementation of its acquistion algorithm", Journal of Beijing University of Aeronautics and Astronautics, vol. 32, no. 1, pp. 53-56, 2006 